\title{
Chemistry of Materials (Article)
}

\section{Supporting Information}

\section{Title}

Functionalizable Organic Nanochannels Based on Lipid Nanotubes: Encapsulation and Nanofluidic Behavior of Biomacromolecules

\section{Authors}

Naohiro Kameta, Mitsutoshi Masuda*, Hiroyuki Minamikawa, Yumiko Mishima, Ichiro Yamashita, and Toshimi Shimizu*

\section{$\underline{\text { Contents }}$}

I. Dynamic light scattering measurement for the QSY7-ferritin

II. STEM image for a NBD-nanotube and TEM image for the NBD-nanotube encapsulating QSY7-ferritin

III. Preparation of QSY7-nanogold

IV. Encapsulation and nanofluidic abilities of the NBD-nanotube toward the QSY7-nanogold and QSY7

V. Determination of diffusion constant for the QSY7-nanogold and QSY7 in the NBD-nanotube channels

VI. Investigation for the encapsulation ability of the glycolipid nanotubes self-assembled from 3 toward ferritin and Dps

VII. Confocal laser scan microscopic, AFM, and TEM images of the N-nanotubes

VIII. XRD and IR spectra of the N-nanotube and helical coil

IX. Estimated molecular packing of the N-nanotube and helical coil 


\section{Dynamic light scattering measurement for the QSY7-ferritin}

Dynamic light scattering (DLS) measurement showed that the QSY7-ferritin has $14.7 \mathrm{~nm}$ diameter (Fig. S1) and negatively charged surface (Fig. S2) at neutral pH.

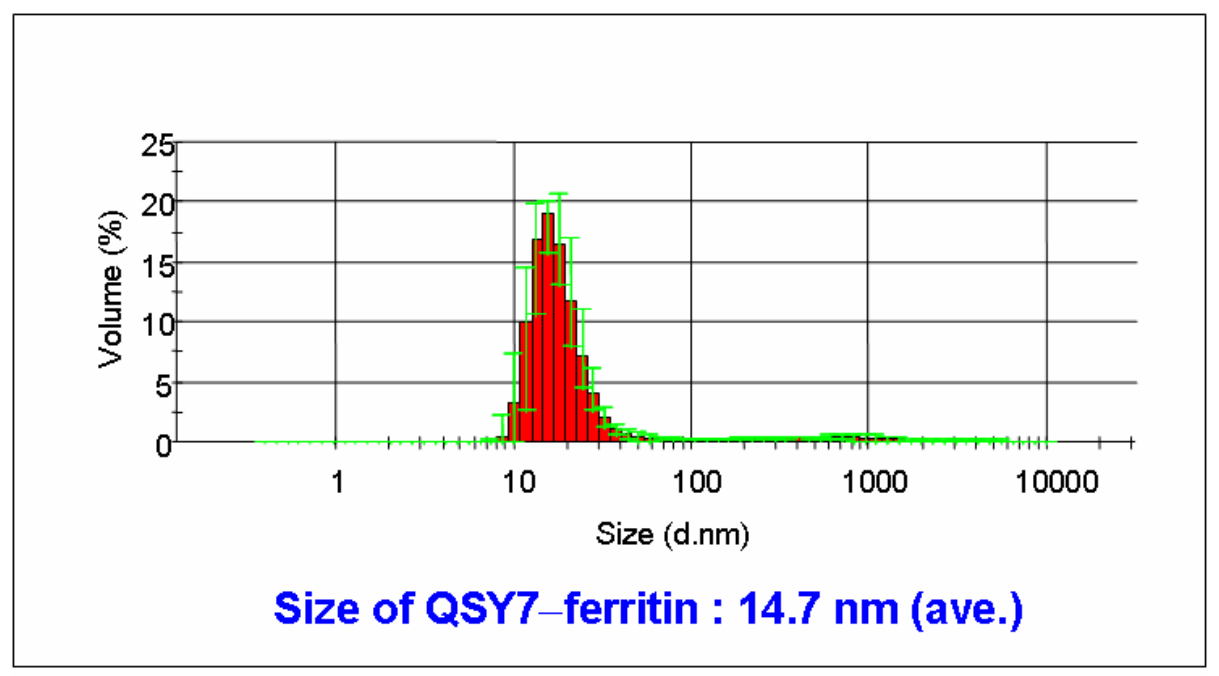

Figure S1. Size distribution of the QSY7-ferritin obtained from DLS measurement.

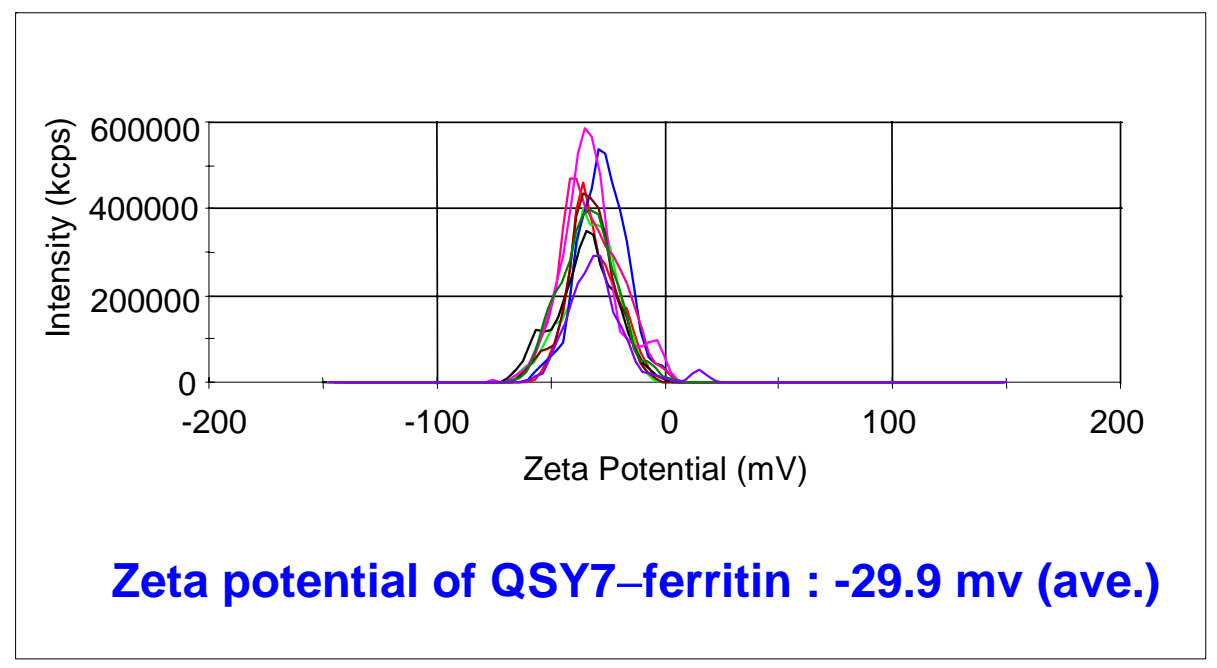

Figure S2. $\zeta$-potential distribution of the QSY7-ferritin obtained from DLS measurement. 


\section{STEM image for a NBD-nanotube and TEM image for the NBD-nanotube encapsulating QSY7-ferritin}

(a)

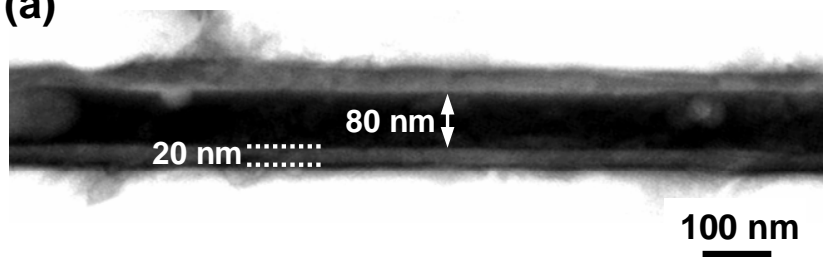

(b)

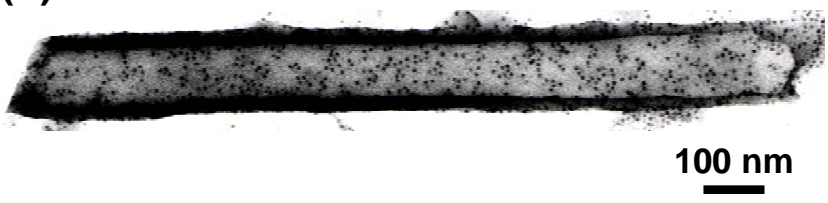

Figure S3. (a) STEM image for a NBD-nanotube. The given hollow cylinder space is imaged in dark by negative staining with phosphotungstate. (b) TEM image for the NBD-nanotube encapsulating QSY7-ferritn in the hollow cylinder. Ferrihydrite cores of the ferritin (7 $\mathrm{nm})$ are shown as small dots located inside the nanotube.

\section{Preparation of QSY7-nanogold}

The QSY7-nanogold was prepared based on known protocols. A methanol solution of $\mathrm{QSY7}^{\circledR}$ carboxylic acid succinimidyl ester (Molecular Probes, $300 \mathrm{nmol}$ ) was added to the aqueous solution containing Monoamino Nanogold ${ }^{\circledR}$ (Nanoprobes, $30 \mathrm{nmol}$ ) $1.4 \mathrm{~nm}$ wide at pH 9 adjusted by $\mathrm{NaOH}$. The mixed solution was stirred for $12 \mathrm{~h}$ at r.t. and then centrifuged (by amicon centriprep, MILLIPORE) to remove low-molecular-weight molecules such as unreacted QSY7 and succinimidyl moiety. 


\section{Encapsulation and nanofluidic abilities of the NBD-nanotube toward QSY7-nanogold and QSY7}

The fluorescence band of the NBD-nanotube completely overlapped with the absorption bands of the QSY7-nanogold and QSY7 as fluorescent acceptors (Fig. S4). Therefore, if these fluorescent acceptors can approach the donor NBD within a few nm on the inner surface of the nanotube, the fluorescence resonance energy transfer (FRET) should occur from NBD to QSY7 moieties. Actually, the fluorescence band of the NBD-nanotube disappeared upon addition of the QSY7-nanogold or QSY7 as well as the QSY7-ferritin (Fig. S5). These quenching phenomena are ascribable to the FRET from the internal NBD to the QSY7-nanogold or QSY7 encapsulated in the hollow cylinder of the NBD-nanotube.
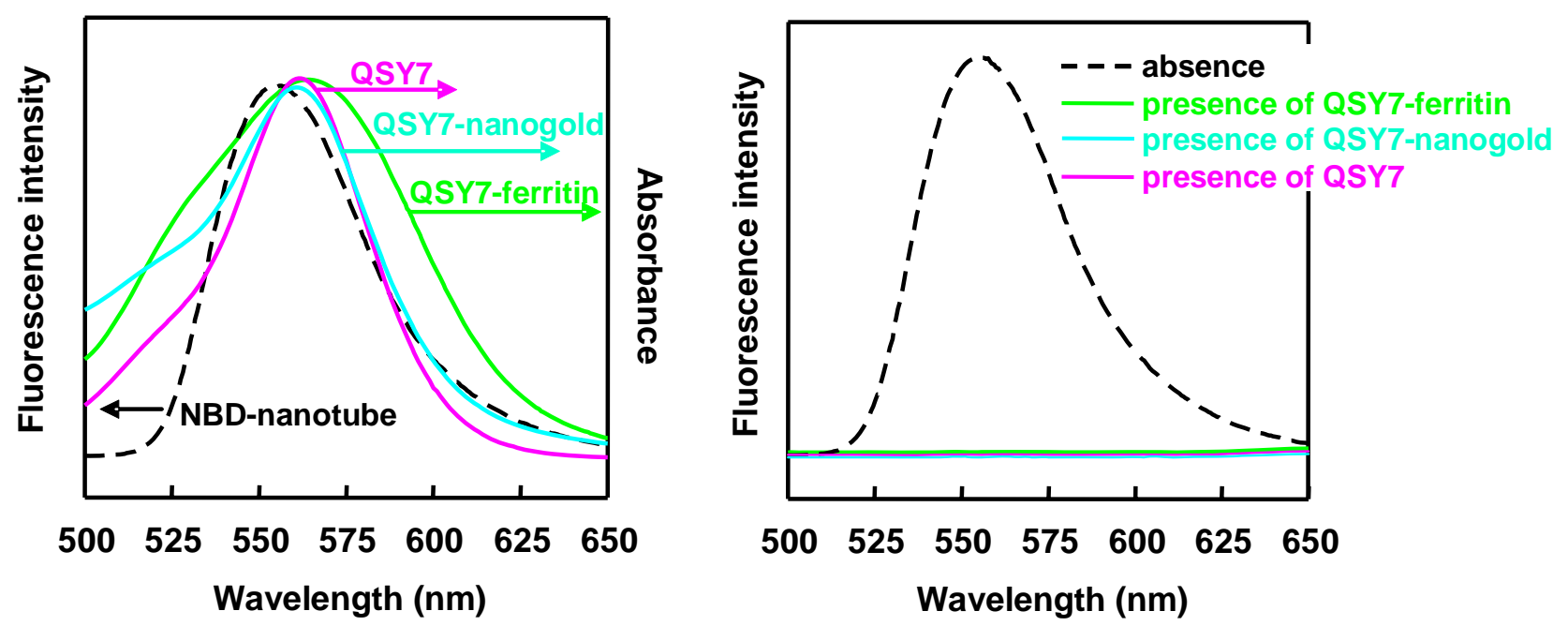

Figure S4 (left). Fluorescent spectrum of the NBD-nanotube and absorption spectra of the QSY7-ferritin, the QSY7-nanogold, and QSY7 in aqueous solutions.

Figure S5 (right). Fluorescent spectra of the NBD-nanotube in the presence and absence of the QSY7-ferritin, the QSY7-nanogold, and QSY7 in aqueous solutions at pH 6.8. 
Time-laps fluorescence microscopic observation visualized the encapsulation and nanofluidic behavior of the QSY7-nanogold and QSY7 in the hollow cylinder of the NBD-nanotube. Upon addition of the QSY7-nanogold or QSY7, both open ends of the NBD-nanotube started to quench (Figs. S6b and S7b). The quenching gradually moved to the central part with laps of time (Figs. S6c-e), and completed in 0.7 and 0.1 sec for the QSY7-nanogolod and QSY7, respectively (Figs. S6f and S7c).
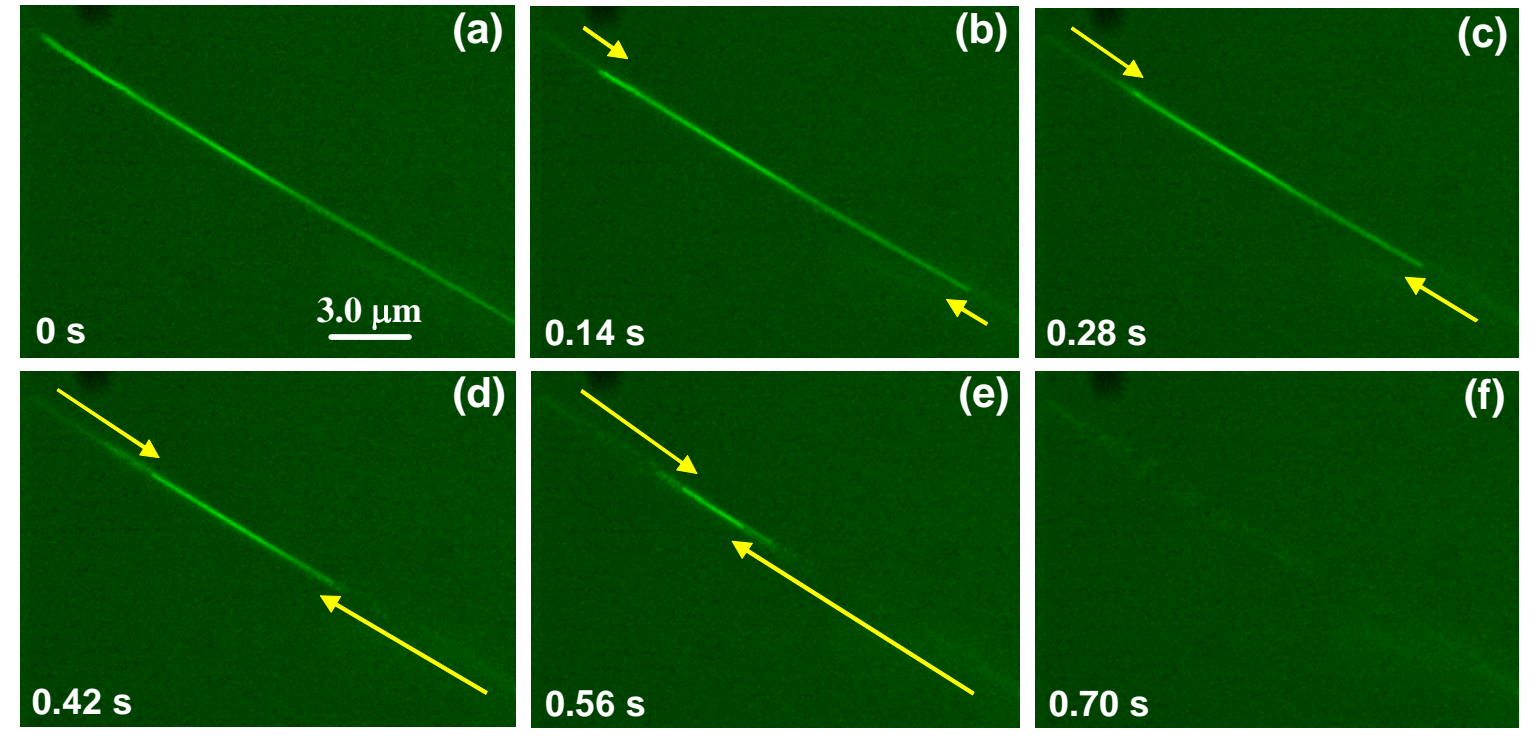

Figure S6. Time-laps fluorescence microscopic images of the NBD-nanotubes upon addition of the QSY7-nanogold. The time course was indicated in the bottom left of each figure.
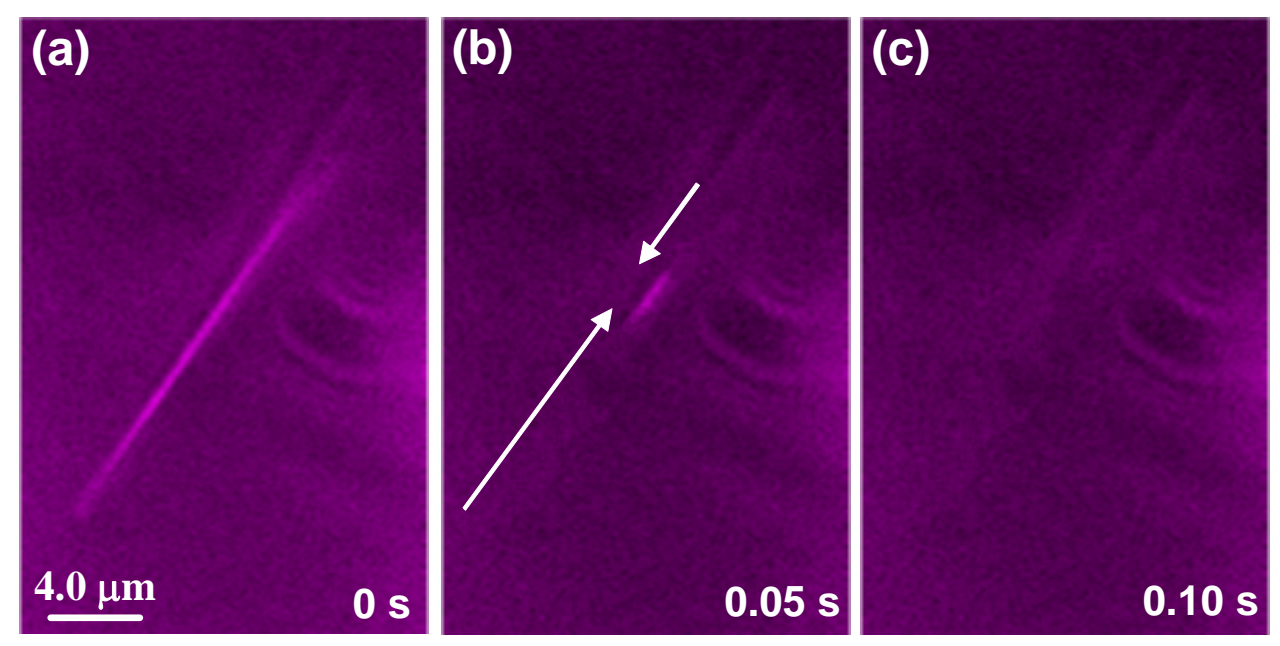

Figure S7. Time-laps fluorescence microscopic images of the NBD-nanotubes upon addition of QSY7. The time course was indicated in the bottom right of each figure. 


\section{Determination of diffusion constant for the QSY7-nanogold and QSY7 in the NBD-nanotube channels}

The square of migration distance $\left(X^{2}\right)$ for the QSY7-nanogold was plotted against the time ( $\Delta t$ ) required for migration on the basis of the above time-laps images (Fig. S8). The obtained linear plot for the QSY7-nanogold encapsulated from the right open end of the NBD-nanotube, as illustrated in Fig. S6, is clearly compatible with the diffusion process. The slope analysis using the equation, $\left[X^{2}\right]$ $=4 D \Delta t$, gave a diffusion constant $D, 6.2 \times 10^{-11} \mathrm{~m}^{2} \mathrm{~s}^{-1}$. The $D$ value is 9 times bigger than that of the QSY7-ferritin $\left(0.7 \times 10^{-11} \mathrm{~m}^{2} \mathrm{~s}^{-1}\right)$. This is ascribable to the difference in the particle size $\left(R_{H}\right)$ between the QSY7-nanogold $(1.4 \mathrm{~nm})$ and the QSY7-ferritin $(14.7 \mathrm{~nm})$ as expected from Einstein-Stokes equation, $D=k T / 6 \eta \pi R_{H}$, where $k, T$, and $\eta$ denote Boltzmann constant, temperature, and viscosity of water, respectively.

We were unable to determine the diffusion constant of QSY7 by time-laps images because of faster diffusion process of molecule.

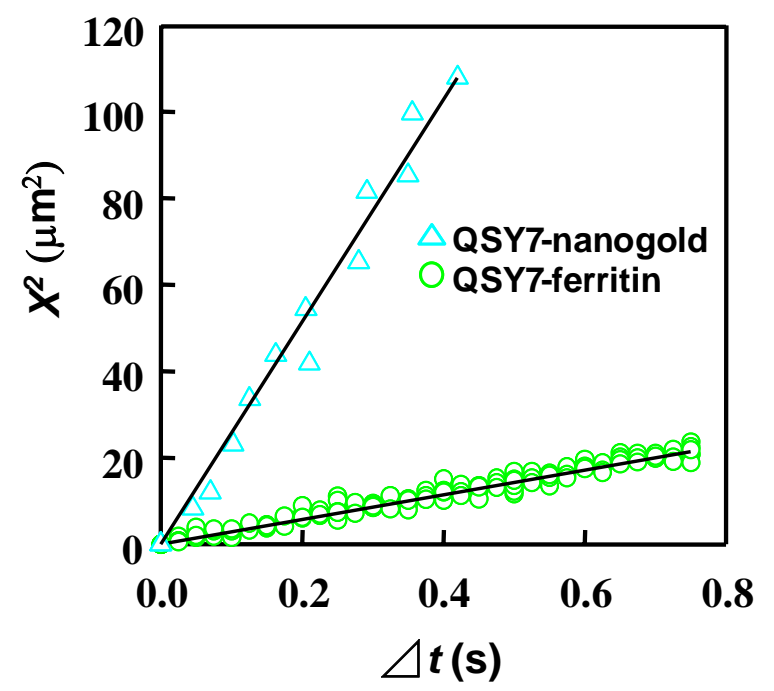

Figure S8. Relationship between the square of migration distance $\left(X^{2}\right)$ of the QSY7-nanogold and QSY7-ferritin in the hollow cylinder of the NBD-nanotube and the time $(\Delta t)$ required. 
VI. Investigation for the encapsulation ability of the glycolipid nanotube self-assembled from 3 toward ferritin and Dps

(a)

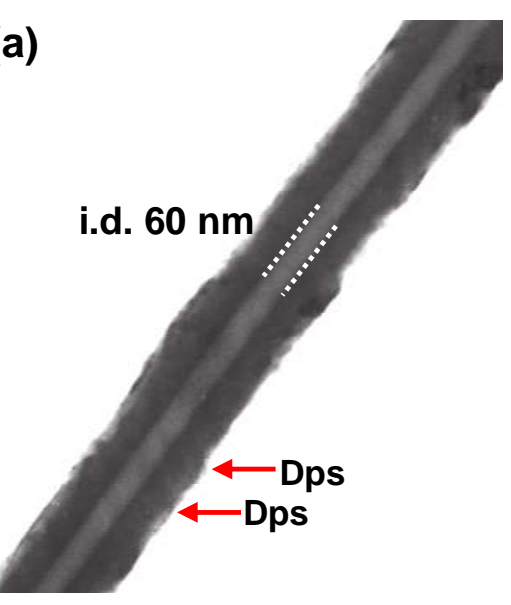

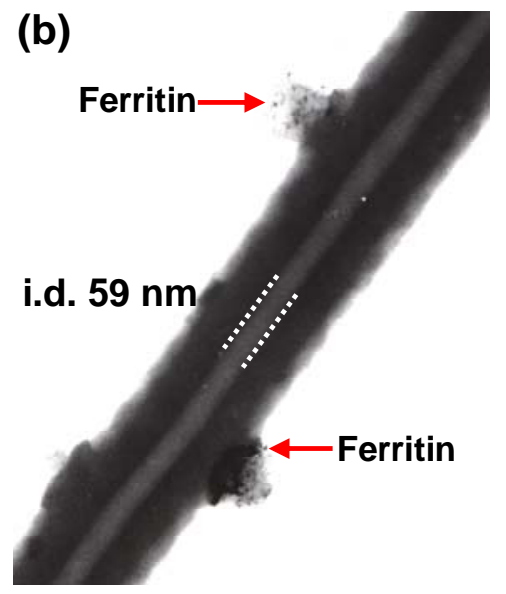

Figure S9. TEM images of the glycolipid nanotube showing no encapsulation ability toward ferritin and Dps. Ferritin and Dps locate at outside of the nanotube.

\section{Confocal laser scan microscopic, AFM, and TEM images of the N-nanotubes}

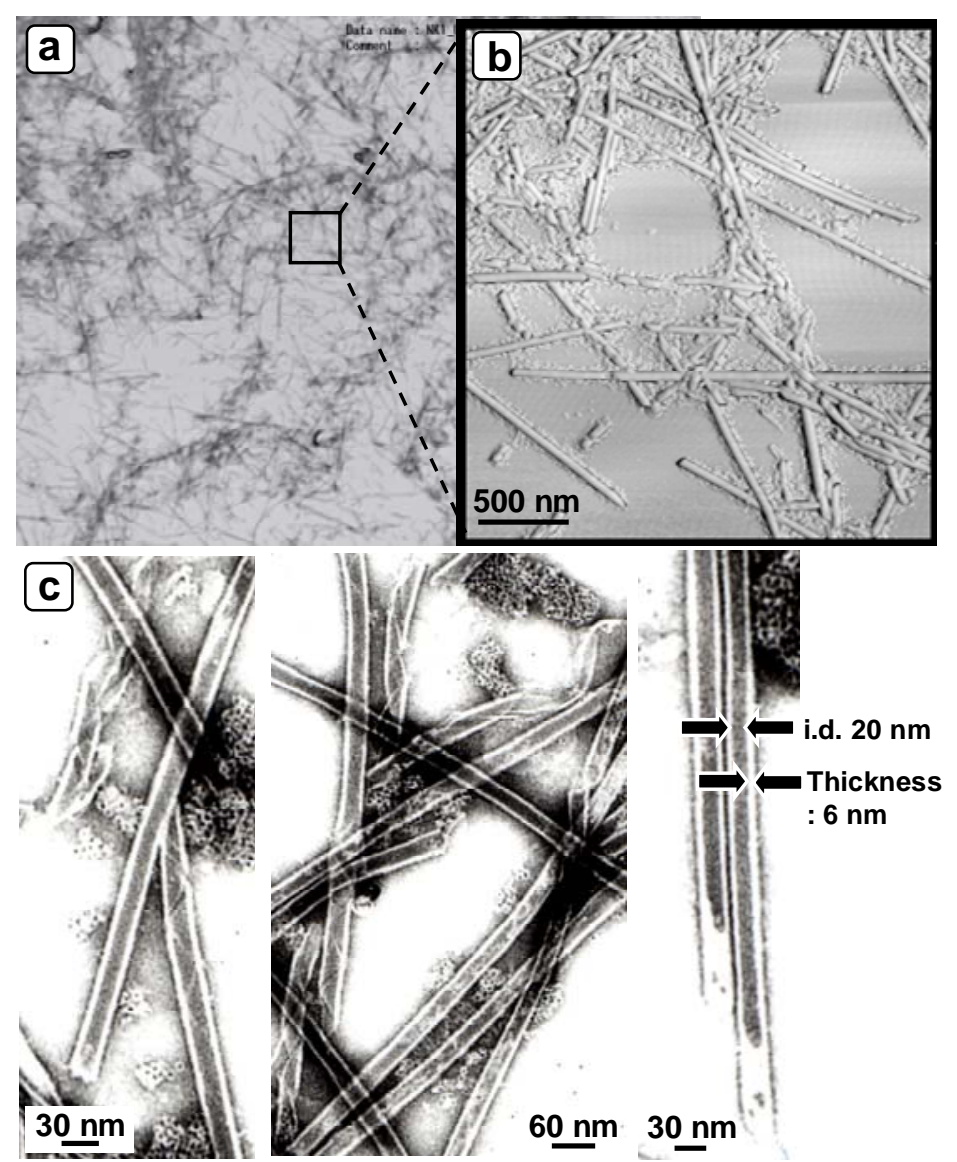

Figure S10. (a) Confocal laser scan microscopic image of $\mathrm{N}$-nanotubes. (b) AFM phase contrast image of the $\mathrm{N}$-nanotubes. (c) TEM images of the $\mathrm{N}$-nanotubes negatively stained by phosphotungstate. 


\section{XRD and IR spectra of the N-nanotube and helical coil}

XRD patterns and the IR spectrum of the $\mathrm{W}$-nanotubes and tapes, self-assembled from the identical bolaamphiphile 1 at pH 10 and 4, respectively, are also shown as references of the MLMs types. The W-nanotubes and tapes consist of the unsymmetrical MLMs with parallel molecular packing and the symmetrical MLMs with antiparallel one, respectively.

(a) Tape

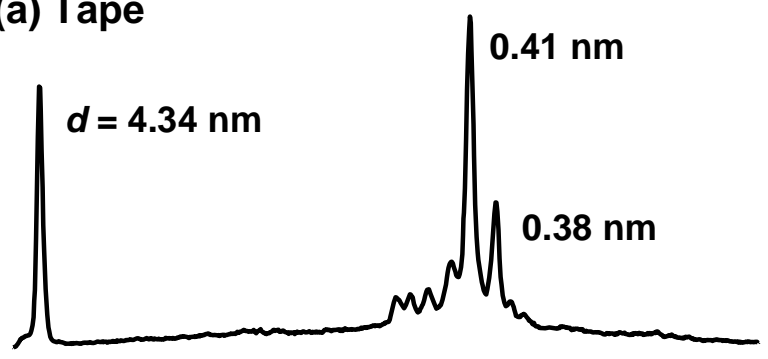

(b) Helical coil

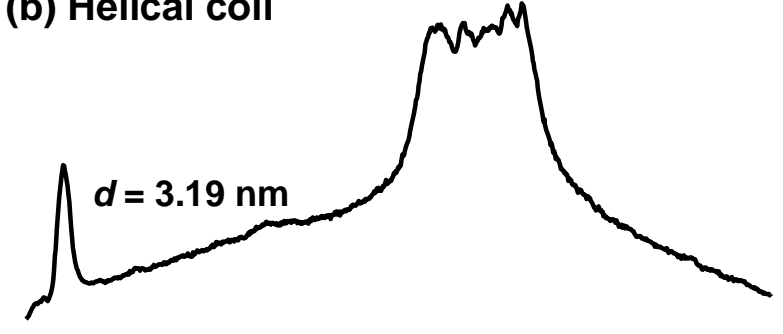

(c) N-Nanotube

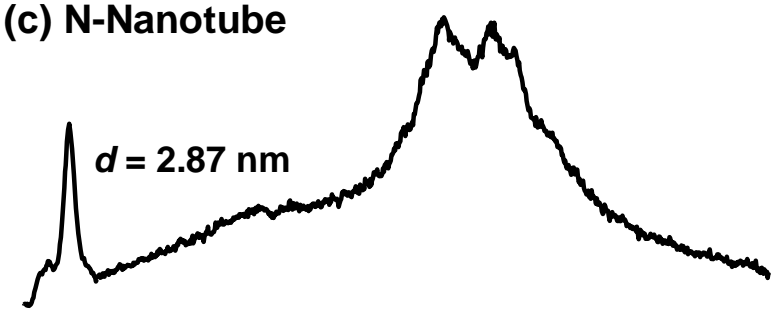

(d) W-Nanotube

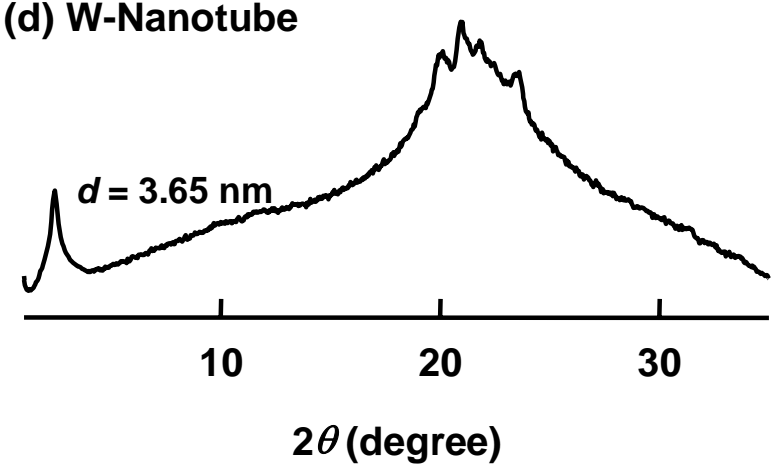

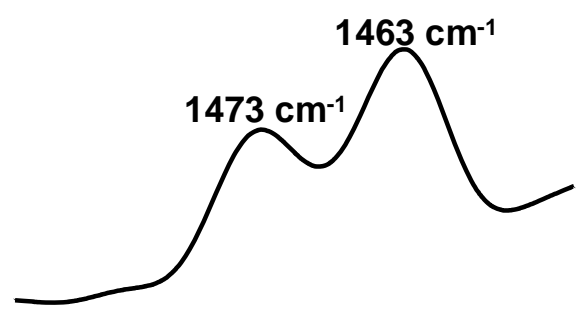
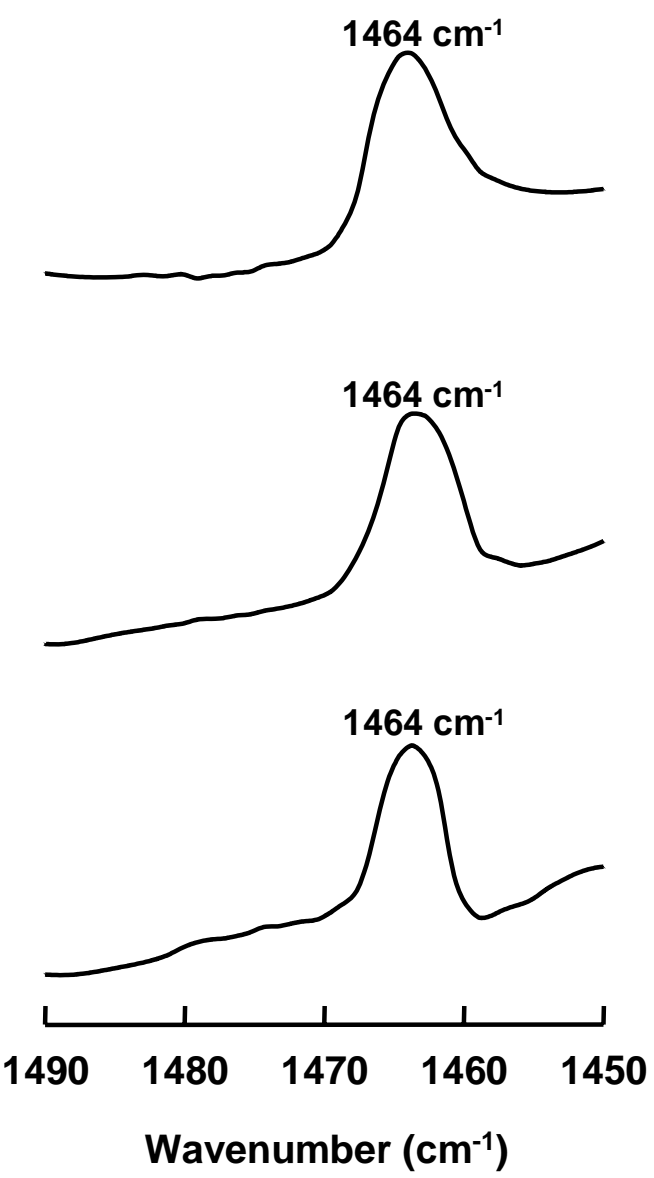

Figure S11. XRD patterns (left) and the IR $\mathrm{CH}_{2}$ scissoring band (right) of the a) tape, b) helical coil, c) N-nanotube, and d) W-nanotube. 


\section{Estimated molecular packing of the N-nanotube and helical coil}

Estimated molecular packing of the W-nanotube and tape, self-assembled from the identical bolaamphiphile $\mathbf{1}$ at pH 10 and 4, respectively, are also shown as references.

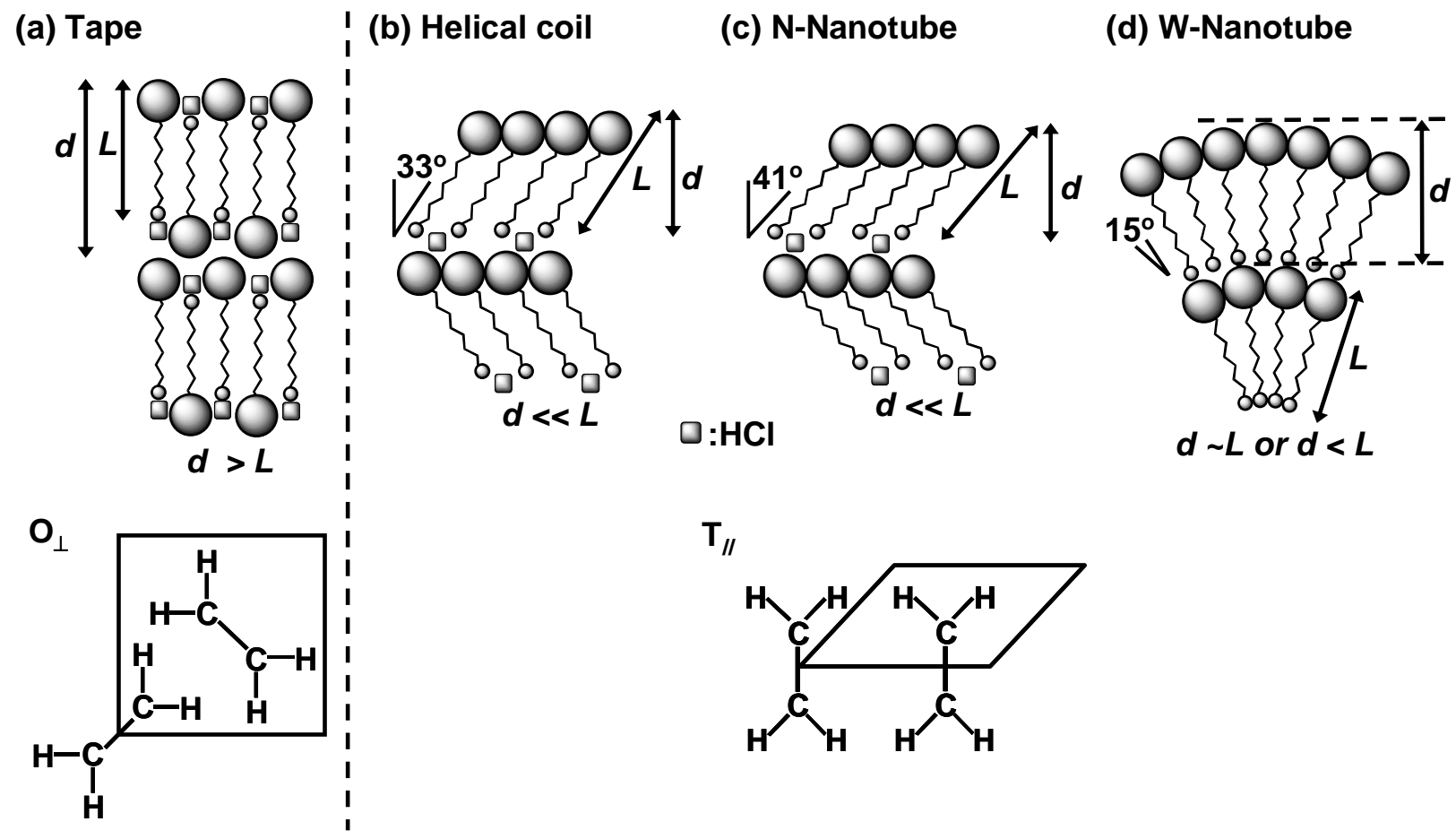

Figure S12. Molecular packing and subcell structure of the oligomethylene spacer in the (a) tape, (b) helical coil, (c) N-nanotube, and (d) W-nanotube. The molecular tilt angle against the each MLM was evaluated on the basis of each $d$ and $L$ value.

Tabel 1. XRD and IR Data for the Molecular Packing of Each Self-Assembly Formed Under Different pH Conditions

\begin{tabular}{ccccc}
\hline XRD, IR data & $\mathrm{pH} 4$ & $\mathrm{pH} 6$ & $\mathrm{pH} 6$ (after aging) & $\mathrm{pH} 10$ \\
\hline morphology & tape & helical coil & N-nanotube & W-nanotube \\
$d(\mathrm{~nm})$ & 4.34 & 3.18 & 2.87 & 3.65 \\
$d$ vs. $L^{a}$ & $d>L$ & $d<<L$ & $d<L$ & $d \sim L$ or $d<L$ \\
$\mathrm{MLM}^{2}$ type & symmetrical & unsymmetrical & unsymmetrical & unsymmetrical \\
$\delta\left(\mathrm{CH}_{2}\right)\left(\mathrm{cm}^{-1}\right)$ & 1473,1463 & $1464(8.6)^{\mathrm{b}}$ & $1464(7.1)^{\mathrm{b}}$ & $1464(5.8)^{\mathrm{b}}$ \\
subcell type & $\mathrm{O}_{\perp}$ & $\mathrm{T}_{/ /}$or $\mathrm{M}_{/ /}$ & $\mathrm{T}_{/ /}$or $\mathrm{M}_{/ /}$ & $\mathrm{T}_{/ /}$or $\mathrm{M}_{/ /}$ \\
molecular tilt $\left(^{\circ}\right)$ & - & 33 & 41 & 15 \\
\hline
\end{tabular}

a) $L$ : Molecular length of $1(3.78 \mathrm{~nm})$

b) Full width at half maximum of the $\mathrm{CH}_{2}$ scissoring band 